\title{
Generation of porous particle structures using the void expansion method
}

\section{Journal Article}

Author(s):

Schenker, Iwan; Filser, Frank T.; Herrmann, Hans J.; Gauckler, Ludwig J.

Publication date:

2009

Permanent link:

https://doi.org/10.3929/ethz-b-000019059

Rights / license:

In Copyright - Non-Commercial Use Permitted

Originally published in:

Granular Matter 11(3), https://doi.org/10.1007/s10035-009-0129-3 


\title{
Generation of porous particle structures using the void expansion method
}

\author{
Iwan Schenker • Frank T. Filser • Hans J. Herrmann • \\ Ludwig J. Gauckler
}

Received: 29 June 2008 / Published online: 13 February 2009

(c) Springer-Verlag 2009

\begin{abstract}
The newly developed "void expansion method" allows for an efficient generation of porous packings of spherical particles over a wide range of volume fractions using the discrete element method. Particles are randomly placed under addition of much smaller "void-particles". Then, the void-particle radius is increased repeatedly, thereby rearranging the structural particles until formation of a dense particle packing. The structural particles' mean coordination number was used to characterize the evolving microstructures. At some void radius, a transition from an initially low to a higher mean coordination number is found, which was used to characterize the influence of the various simulation parameters. For structural and void-particle stiffnesses of the same order of magnitude, the transition is found at constant total volume fraction slightly below the random close packing limit. For decreasing void-particle stiffness the transition is shifted towards a smaller void-particle radius and becomes smoother.
\end{abstract}

Keywords Colloid - Coordination number - Discrete element method · Microstructure generation · Porosity

\section{Introduction}

Mechanical tests on coagulated colloids have shown that the local arrangement of the colloidal particles has a strong influence on the macroscopic mechanical properties. Colloids

I. Schenker $(\bowtie) \cdot$ F. T. Filser · L. J. Gauckler

Department of Materials, Institute of Nonmetallic Materials, ETH Zurich, 8093 Zurich, Switzerland

e-mail: iwan.schenker@mat.ethz.ch

\section{H. J. Herrmann}

Computational Physics for Engineering Materials, Institute for Building Materials,

ETH Zurich, 8093 Zurich, Switzerland with a more "heterogeneous" microstructure possess up to one order of magnitude higher elastic modulii and yield strengths than their "homogeneous" counterparts [1].

Experimentally, colloidal microstructures with different degrees of heterogeneity are obtained using an internal gelation method (DCC direct coagulation casting [2,3]). The method allows for an in situ, i.e. undisturbed, transition of the inter-particle potential from repulsive to attractive. There are two principal pathways leading to different microstructures: changing the $\mathrm{pH}$ of the suspension ( $\Delta \mathrm{pH}$-method) or increasing the ionic strength in the suspension ( $\Delta \mathrm{I}$-method). The first pathway shifts the $\mathrm{pH}$ to the particles' isoelectric point and produces more "homogeneous" microstructures through diffusion limited aggregation. In the second pathway the ionic strength in the suspension is increased at a constant $\mathrm{pH}$ which compresses the Debye length of the repulsive potential leading to more "heterogeneous" microstructures due to reaction rate limited aggregation of the particles [4].

Alternatively, heterogeneous microstructures can as well be obtained by $\Delta \mathrm{pH}$-destabilization in conjunction with small amounts of alkali-swellable polymer particles (ASP), $80 \mathrm{~nm}$ in diameter in the unswollen state [5]. The ASP particles were admixed to the structural particles of $200 \mathrm{~nm}$ in diameter under acidic conditions and swelled upon increasing $\mathrm{pH}$ during the internal gelling reaction of the DCC process unfolding to $800 \mathrm{~nm}$ in diameter, and thus pushing the structural particles in their vicinity. Thereby, larger pores and thus more heterogeneous microstructures are produced. Those samples with ASP exhibit much higher mechanical properties than samples without ASP. In particular, ASP samples present comparably high mechanical properties as samples with heterogeneous microstructures produced by the $\Delta \mathrm{I}$-method.

These experimental findings suggest that the colloid's microstructure strongly determines its macroscopic mechanical 
properties. The relation between structure and mechanical properties, however, is not yet understood. One way to look at this question is by computational means using simulation techniques such as the discrete element method (DEM). This method takes into account the particulate nature of a colloid and allows for an investigation of the force distribution inside the particle network during deformation as a function of the colloid's microstructure. However, this method needs to be supplied with initial particle configurations. In preceding works, Brownian dynamics simulation (BD) was used to study the coagulation dynamics and the evolving microstructures in colloidal suspensions [6,7]. These simulations were based on physical laws and widely accepted theories such as the Stoke's drag force, Brownian motion and the DLVOtheory [8], describing the inter-particle potential. The resulting microstructures agree well with experiments [9] and can be used as initial particle configurations for further DEM simulations to establish the link between microstructure and macroscopic mechanical properties. However, the BD method requires evaluating complex equations at each time-step in order to determine the various forces acting on the particles. Therefore, it is time-consuming, especially in the case of a repulsive energy barrier and moreover, structures with volume fractions exceeding 0.4 have not yet been simulated. For processing reasons, ceramic engineers are interested in preferably high solid's phase volume fractions and in particular in volume fractions exceeding 0.4 .

Inspired by the generation of heterogeneous micro-structures using ASP we developed the void expansion method (VEM), which allows for a fast and efficient computational generation of porous microstructures over a broad range of volume fractions and especially those exceeding 0.4 .

In this publication, VEM is presented and the influence of various simulation parameters such as the system size, the number of particles within the system or the elastic properties of the particles on the development of the mean coordination number is analyzed for a wide range of volume fractions between 0.2 and 0.55 .

\section{Materials and methods}

\subsection{Discrete element method}

The void expansion method is implemented using DEM [10] and in particular, the particle flow code in three dimensions $\left(\mathrm{PFC}^{3 \mathrm{D}}\right)$ from Itasca Consulting Group, Inc., Minneapolis, Minnesota, USA [11] is used. DEM is an iterative method in which discrete spherical particles are used to build up more complex structures. At each point in time the forces on each particle are calculated. The time-step is chosen small enough to assume a constant force during the time-step, which allows for the linearization of the equations of motion enabling an efficient calculation of the particles' next positions and velocities.

The forces on the particles included in our model arise from a linear elastic contact law between the particles and damping. In particular, no other forces such as long range forces between particles or gravity are considered. PFC ${ }^{3 \mathrm{D}}$ uses a soft-contact approach, where the rigid particles are allowed to overlap at contact points. The contact law relates the forces acting on two contacting particles, in our case, linearly to the relative displacement between these particles. The magnitude of the normal contact force $F_{n}$ is given by Eq. (1)

$F_{n}=k_{n} U_{n}$

where $k_{n}$ denotes the normal stiffness and $U_{n}$ the overlap. The shear stiffness $k_{s}$ relates an incremental displacement in shear direction $\Delta U_{s}$ to the shear contact force $\Delta F_{S}$ via Eq. (2).

$\Delta F_{s}=k_{s} \Delta U_{s}$

The linear elastic contact law is thus parameterized by its normal and its shear particle stiffness.

Energy dissipation is introduced via a local damping term similar to that described by Cundall [12]. The damping force, characterized by its damping coefficient $d$, is added to the equations of motion and is proportional to the force acting on the particle. Thereby, only accelerating motion is damped and the direction of the damping force is opposed to the particle's velocity [11].

Thus, the forces in our model are characterized by three microscopic parameters: the particle's normal stiffness, its shear stiffness and the damping coefficient. In this work the inter-particle friction coefficient was set to zero in order to allow the maximum particle rearrangement during the void expansion.

\subsection{Void expansion method}

The void expansion method relies on two distinct kinds of particles: "structural particles" that constitute the final microstructure and "void-particles" that are only used during the generation of the structure. For clarity purposes, the first ones will be referred to as structural particles or simply particles and the latter ones will explicitly be termed void-particles throughout this publication. The physically relevant macroscopic parameters characterizing the final microstructures are $N_{S}$, the number of structural particles, $r_{S}$, their radius and $\Phi_{S}$, the volume fraction of the structural particles. Using these parameters the edge length $l$ of the cubic simulation box with periodic boundary conditions is calculated using Eq. (3).

$l=r_{S}\left(\frac{4 N_{S} \pi}{3 \Phi_{S}}\right)^{1 / 3}$ 
The $N_{S}$ particles are randomly placed in the simulation box with an initial particle radius of $r_{S} /(m+1)$, thus $(m+1)$ times smaller than the final $r_{S}$, with $m$ being the number of subsequent radius blow-up steps. We use $m=10$ in our simulations. At each blow-up step, the initial particle radius is added to the current particle radius, followed by an equilibration of the structure, until, after the $m$ th step, the final particle radius $r_{S}$ is reached. This cyclic growing of the particles is needed in order to achieve volume fractions higher than approximately 0.35 without a considerable particle overlap which represents high local stresses.

In addition to the structural particles, $N_{V}$ void-particles with an initial radius $r_{V} \ll r_{S}$ are randomly placed in the simulation box. We used $r_{V}=0.005 r_{S}$. After the structural particles have reached their final size the radius of the voidparticles is increased cyclically. At each cycle their initial radius is added to their current radius, thereby simulating the swelling of the ASP. After each incremental increase of the void-particle radius, relaxation steps are performed in order to equilibrate the microstructure. This iterative procedure is repeated until the structural and the void-particles are densely packed and any further increase of the void-particle size leads to a compaction of the particles, which is reflected by an increase of the strain energy inside the microstructure. Before each increase of the void particle radius the positions of the structural particles are stored, which allows for a subsequent analysis of the microstructure as function of pore size, i.e. the void-particle's radius.

In this study, the mean coordination number $C N$ of the structural particles alone is used to characterize the evolving microstructures during the expansion of the void-particles. In particular, the coordination number of a structural particle is given by the number of neighboring structural particles with a separation distance smaller than $d_{\epsilon}=(1+\epsilon) 2 r_{S}$, with $\epsilon=0.01$.

The density of bulk alumina was taken for the density of the structural particles $\rho_{S}$. The void-particle density $\rho_{V}$ was set to a ten times smaller value $\rho_{V}=\rho_{S} / 10$ in order to reduce the inertia of the void-particles. Table 1 compiles the simulation parameters used in this work.

\section{Results and discussion}

In this study, the mean coordination number $C N$ is used to characterize the evolving microstructures during the expansion of the void-particles. The evolution of $C N$ as function of the void- to structural particle radius ratio $q=r_{V} / r_{S}$ is shown in Fig. 1 using 8,000 structural particles, 2,000 voidparticles and a volume fraction of 0.4. Also, the conventions of the nomenclatures used throughout this paper are shown in this figure.
Table 1 Simulation parameters

\begin{tabular}{lll}
\hline Parameter & Symbol & Value \\
\hline Number of particles & $N_{S}$ & $4,000,8,000$ \\
Particle radius & $r_{S}$ & $2.5 \times 10^{-7} \mathrm{~m}$ \\
Normal structural particle stiffness & $k_{n, S}$ & $10^{2}, 10^{3} \mathrm{~N} / \mathrm{m}$ \\
Shear structural particle stiffness & $k_{s, S}$ & $10^{-3}, 10^{-2} \mathrm{~N} / \mathrm{m}$ \\
Number of void-particles & $N_{V}$ & $400-16,000$ \\
Normal void-particle stiffness & $k_{n, V}$ & $10^{-5}$ to $10^{3} \mathrm{~N} / \mathrm{m}$ \\
Shear void-particle stiffness & $k_{S, V}$ & $10^{-9}$ to $10^{-1} \mathrm{~N} / \mathrm{m}$ \\
Damping coefficient & $d$ & 0.7 \\
Volume fraction & $\Phi_{S}$ & $0.2-0.55$ \\
Structural particle density & $\rho_{S}$ & $3,690 \mathrm{~kg} / \mathrm{m}^{3}$ \\
Void-particle density & $\rho_{V}$ & $369 \mathrm{~kg} / \mathrm{m}^{3}$ \\
\hline
\end{tabular}

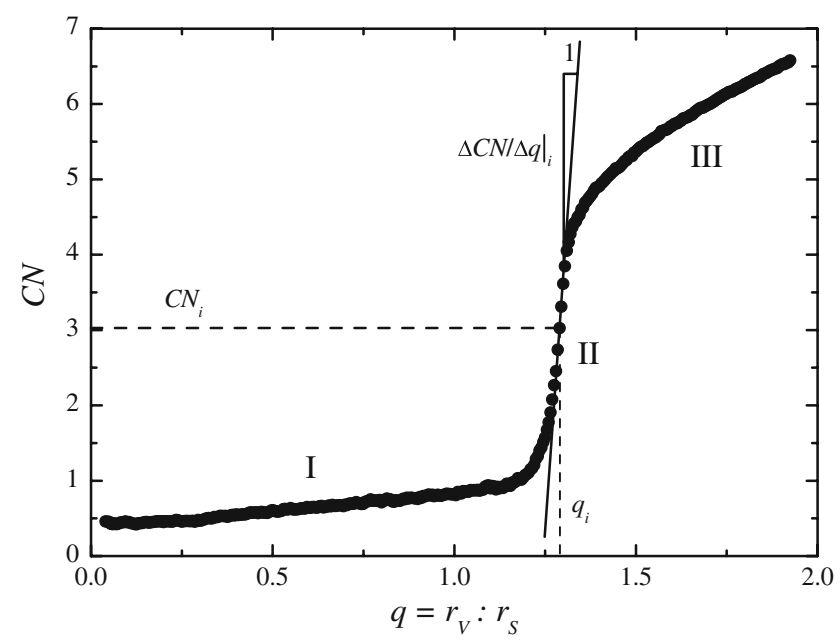

Fig. 1 Mean coordination number $C N$ as a function of the void- to structural particle radius ratio $q$ using 8,000 structural particles, 2,000 void-particles and a volume fraction of 0.4

The curve presents the three distinct regimes typical to all curves analyzed throughout this study (Fig. 1): the initial stage (I) is characterized by a small slope and a low mean coordination number. The slope within the second stage (II) increases drastically and the curve shows an inflection point. In the third stage (III), the particles are densely packed and any further increase of the coordination number is due to the compaction of the particles reflected by a significant increase of the structure's intrinsic strain energy. The transition stage can be interpreted as a phase change between stage I, in which the particles can move freely and stage III, in which the particles' movements are arrested. The inflection point in the transition region (II) is used to characterize the various curves that have been simulated. It is defined by three parameters: the void- to structural particle radius ratio $q_{i}=r_{V, i} / r_{S}$, with $r_{V, i}$ being the void-particle radius at the inflection point, the mean coordination number $C N_{i}$ and the maximum slope, denoted 
by $\Delta C N /\left.\Delta q\right|_{i}$. Further characteristic parameters for the various curves are the void- to structural particle number ratio $n=N_{V} / N_{S}$ and the targeted volume fraction $\Phi_{S}$, which is the volume fraction of the structural particles alone.

In the following, sensitivity analyses show the influence of various VEM simulation parameters, especially the voidand structural particle numbers (Sect. 3.1) and the targeted volume fraction (Sect. 3.2), on $C N$. These simulations use a void-particle normal and shear stiffness of $10^{2}$ and $10^{-2} \mathrm{~N} / \mathrm{m}$, respectively and a structural particle normal and shear stiffness of $10^{3}$ and $10^{-2} \mathrm{~N} / \mathrm{m}$, respectively. In Sect. 3.3 the scaling behavior of $C N$ above the inflection point as function of the total volume fraction is analyzed. The influence of the void-particle stiffness on the evolving microstructures is investigated thereafter in Sect. 3.4 for two distinct structural particle normal stiffnesses: $10^{3}$ and $10^{2} \mathrm{~N} / \mathrm{m}$. The structural particles' normal to shear stiffness ratio was fixed at $10^{5}$.

\subsection{Influence of the void- and structural particle numbers}

Microstructures with a volume fraction $\Phi_{S}=0.4$ have been generated using various numbers of structural and voidparticles, $N_{S}$ and $N_{V}$, respectively. $N_{V}$ essentially regulates the void size. Indeed, the higher $N_{V}$ is chosen, the fewer blow-up steps are necessary in order to densely pack the structural particles. The influence of $N_{V}$ on the VEM was probed with $N_{V}$ ranging between 400 and 16,000 for $N_{S}=$ 8,000 . The size dependency of our system was tested with additional simulations for $N_{S}=4,000$ using 2,000 and 4,000 void-particles.

The simulations show that the evolving microstructures depend on the void- to structural particle number ratio $n$, but they are independent of the individual absolute numbers of $N_{V}$ and $N_{S}$. Increasing $n$ shifts the transition region (II) towards a smaller void- to structural particle radius ratio $q$. Figure 2 shows $q_{i}$ normalized by $n^{-1 / 3}$ as a function of $n$ yielding a constant value given in Eq. (4).

$q_{i} n^{1 / 3}=0.82 \pm 0.01$

The total volume fraction at the inflection point $\Phi_{T, i}$ (void and structural particles) is calculated via Eq. (5).

$\Phi_{T, i}=\Phi_{S}\left(1+q_{i}^{3} n\right)$

Thus, a constant value of $q_{i} n^{1 / 3}$ entails a constant $\Phi_{T, i}$. Indeed, the mean total volume fraction at the inflection point is $62.3 \pm 0.9 \mathrm{vol} . \%$ close to $64 \mathrm{vol} . \%$, the characteristic volume fraction of random close packings (RCP) [13].

Figure 2 further shows the differential increase in coordination number at the inflection point $\Delta C N /\left.\Delta q\right|_{i}$ normalized by $n^{-1 / 3}$ as a function of $n$ yielding a constant value given in Eq. (6).

$\Delta C N / \Delta q{ }_{i} n^{1 / 3}=9.16 \pm 0.68$

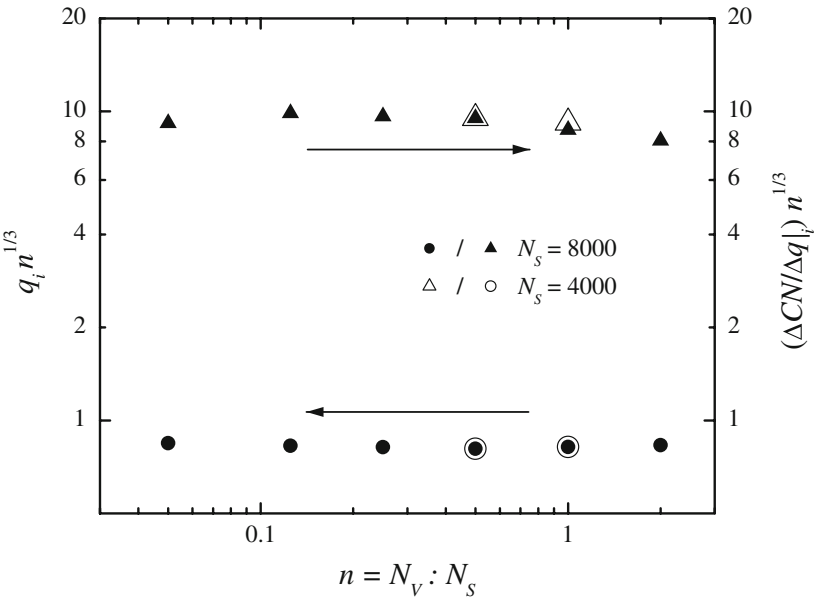

Fig. 2 Void- to structural particle radius ratio $\left(q_{i}\right.$ normalized by $\left.n^{-1 / 3}\right)$ at the inflection point (circles left scale) and the corresponding differential increase in coordination number $\left(\Delta C N /\left.\Delta q\right|_{i}\right.$ normalized by $n^{-1 / 3}$, triangles right scale) as a function of the void- to structural particle number ratio $\left(n=N_{V} / N_{S}\right)$. Open and filled symbols denote simulations with $N_{S}=4,000$ and 8,000 , respectively

The best power law fit of $\Delta C N /\left.\Delta q\right|_{i}$ as a function of $n$ yields an exponent of 0.3 slightly below $1 / 3$ used for normalization in Fig. 2. The fit is very good as indicated by the correlation coefficient $R^{2}=0.98$.

In particular, the use of two distinct numbers of structural particles $\left(N_{S}=4,000\right.$ and 8,000) for two void- to structural particle number ratios $(n=0.5$ and 1.0) shows that the evolution of the mean coordination number is independent of the system size. Virtually identical values for both $q_{i} n^{1 / 3}$ and $\Delta C N / \Delta q{ }_{i} n^{1 / 3}$ as function of $n$ were obtained as shown in Fig. 2.

Combining Eqs. (4) and (6) gives Eq. (7)

$\left.\Delta C N\right|_{i}=\left.11.2 q \Delta q\right|_{i}$

which, after integration, results in Eq. (8)

$C N_{i}=5.6 q_{i}^{2}+K$

with $K$ a constant. Thus, $C N_{i}$ is expected to scale as $q_{i}^{2}$, which means that $C N_{i}$ essentially scales with the surface of the void particles. $C N_{i}$ as a function of $q_{i}$ and its fit (dashed line) are shown in Fig. 3. The trend is reproduced, however, the quality of the fit is rather low $\left(R^{2}=0.76\right)$. In particular, the two data points with $q_{i}$ close to unity present a noticeable deviation of the general trend of increasing $C N_{i}$ for increasing $q_{i}$. This case is remarkable as it corresponds to an approximately monodispersed binary mixture of the structural and void-particles. A more detailed investigation, especially in the region of $q_{i} \approx 1$, would require much more simulation runs spanning a wider range of particle number ratios $n$, which goes beyond this publication's scope. 


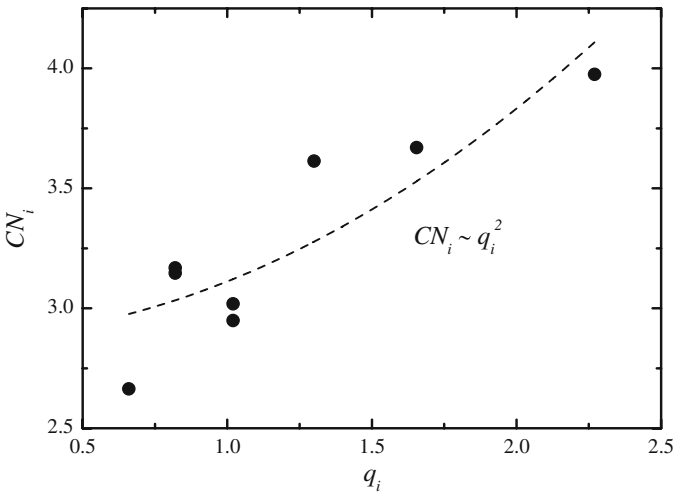

Fig. 3 Mean coordination number $C N_{i}$ as function of the void- to structural particle radius ratio $q_{i}$, both at the inflection point, for various void- to structural particle number ratios $n$

\subsection{Influence of the volume fraction}

For processing reasons of ceramic bodies via colloidal routes engineers are usually interested in volume fractions as high as possible. A minimum of $40 \mathrm{vol}$.\% was required to perform uniaxial compression test on structures fabricated using the DCC process [14]. Furthermore, the volume fraction is easily accessible experimentally and is therefore widely used as a comparative value for various experiments. Thus, the influence of the volume fraction of the structural particles $\Phi_{S}$ was investigated by means of $C N$ as a function of $q$ for $\Phi_{S}$ ranging from 0.2 to 0.55 and for $n=0.5\left(N_{S}=\right.$ 4,000 and $N_{V}=2,000$ ). The total volume fraction at the inflection point $\Phi_{T, i}$ (void- and structural particles) is calculated using Eq. (5). Again, $\Phi_{T, i}$ yields a constant value of $61.5 \pm 0.5 \mathrm{vol} . \%$, slightly below the RCP limit. Thus, $\Phi_{T, i}$ constitutes an upper boundary for $\Phi_{S}$. Indeed, for $\Phi_{S}$ approaching $\Phi_{T, i}$ and $n \neq 0, q_{i}$ approaches zero and any expansion of the void-particles is prohibited.

Figure 4 shows that $C N_{i}$ increases with increasing volume fraction $\Phi_{S}$. As shown above, $\Phi_{T, i}$ is constant for $\Phi_{S}$ ranging from 0.2 to 0.55 , which indicates that at the inflection point, the structural particles are equally dense packed for all $\Phi_{S}$. Hence, $C N_{i}$ may be expected to be a constant value, which seems to contradict Fig. 4. Plausibility considerations based on geometry give a possible explanation for this $\Phi_{S}$-dependence of $C N_{i}$. The sum of $\Phi_{S}$ and $\Phi_{V, i}$ equals $\Phi_{T, i}$, and is constant. Hence, rising $\Phi_{S}$ results in a lower $\Phi_{V, i}$ and vice versa. Because $N_{V}$ is constant, $\Phi_{V, i}$ only changes by the variation of $r_{V, i}$. An increasing $\Phi_{V, i}$ entails an increase in $r_{V, i}$, and vice versa. Structural particles in contact with void-particles have a lower coordination number than those which are only surrounded by other structural particles because only contacts between structural particles are considered by definition of $C N$. The number of structural particles in contact with void-particles scales essentially with the total surface of the void-particles. The smaller the total

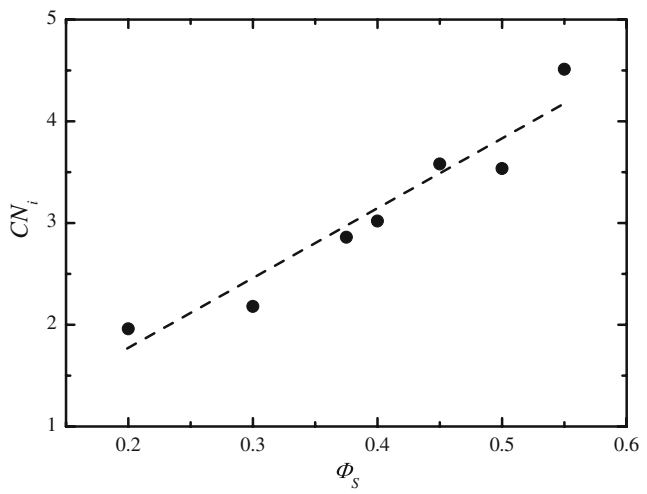

Fig. 4 Mean coordination number at the inflection point $C N_{i}$ as a function of the volume fraction of the structural particles $\Phi_{S}$

void surface, the less contacts between structural and voidparticles exist and the more structural particles are only surrounded by other structural particles. Hence, increasing $\Phi_{S}$ results in increasing $C N_{i}$.

Similar considerations were used by Kruyt and Rothenburg [15] who found a linear dependence between a particle's coordination number and its radius in the case of two-dimensional assemblies of polydisperse particles. Further studies are necessary to confirm a linear dependence between $C N_{i}$ and $\Phi_{S}$ as suggested by the line in Fig. 4 .

\subsection{Scaling behavior of $C N$}

In order to analyze the scaling behavior of $C N$ above $C N_{i}$ (region III) as a function of the total volume fraction $\Phi_{T}$ the various curves analyzed in Sects. 3.1 and 3.2 were fitted using a power law given in Eq. (9)

$\left(C N-C N_{i}\right) \propto\left(\Phi_{T}-\Phi_{T, i}\right)^{\beta}$

with the exponent $\beta$ as fit parameter. A selection of these curves is presented in Fig. 5 for simulations as function of the void- to structural particle number ratio $n$ (circles) and as function of the volume fraction of the structural particles $\Phi_{S}$ (triangles). The corresponding power law fits are shown as lines.

For the simulations with varying $n$, an exponent $\beta_{n}=$ $0.39 \pm 0.04$ was obtained. The simulations in dependence of $\Phi_{S}$ yield $\beta_{\phi_{S}}=0.35 \pm 0.03$. For all fits a very high correlation coefficient $R^{2}>0.999$ was achieved indicating excellent fits. In particular, our simulations suggest that the exponents $\beta$ is independent of $n$ and $\Phi_{S}$. The average over all simulations results in an exponent $\beta=0.37 \pm 0.04$. This exponent is below the value 0.5 found in literature $[16,17]$, where however dense instead of porous structures were considered. 


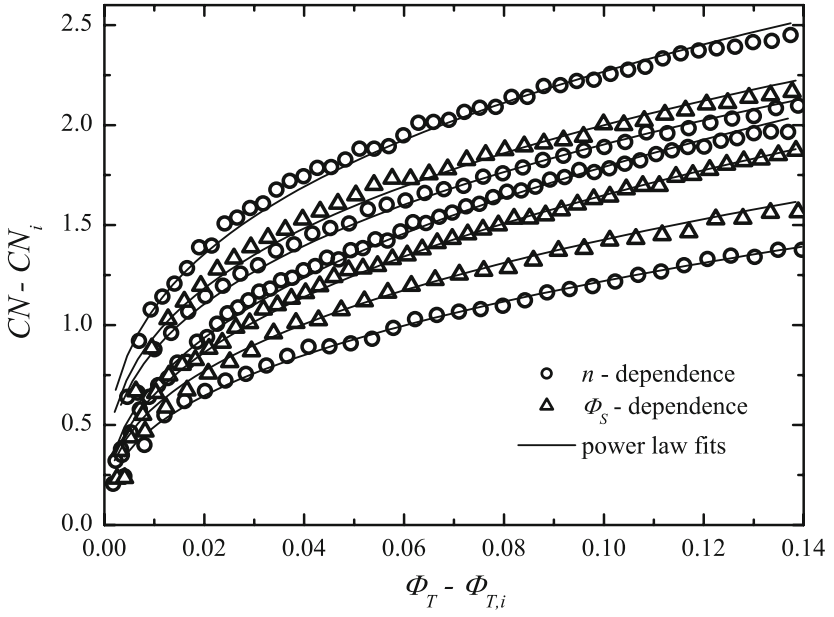

Fig. 5 Scaling behavior of the coordination number $C N$ above the inflection point in dependence of the total volume fraction $\Phi_{T}$ for various curves (open symbols) and corresponding power law fits (lines)

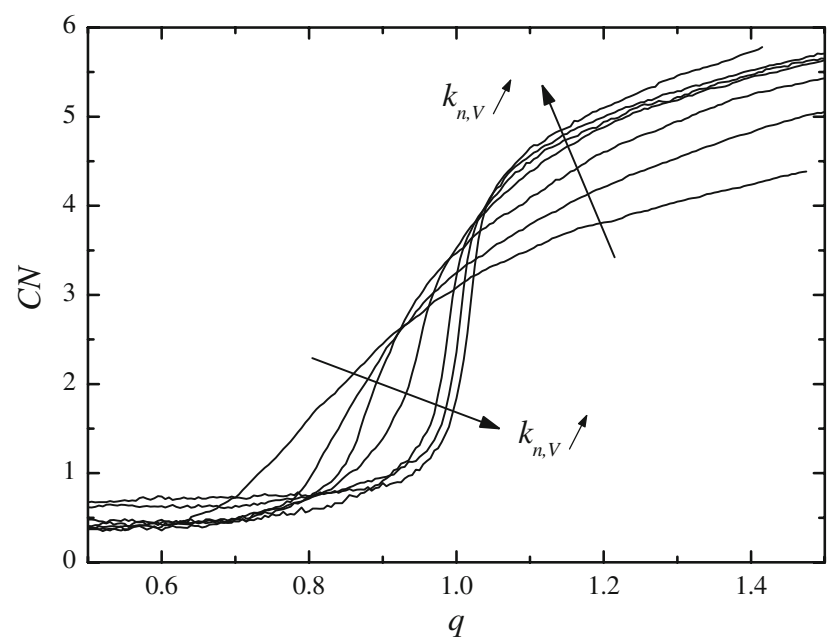

Fig. 6 Mean coordination number $C N$ as a function of the void- to structural particle radius ratio $q$ for a void- to structural particle number ratio $n=0.5$ and for various void-particle stiffnesses. The arrows indicate the direction of increasing void-particle stiffness

\subsection{Influence of the void-particle stiffness}

The void-particle stiffness essentially regulates the extent of the overlap of a void-particle with structural particles or other void-particles. Indeed, for a constant compressive force, a lower void-particle stiffness allows for larger overlaps between a void-particle and its neighbors. Thus, the void-particle stiffness is an important parameter for the microstructure and its evolution. For this investigation, the void-particle normal to shear stiffness ratio $k_{n, V} / k_{s, V}$ is kept constant at $10^{4}$, $n$ was fixed at 0.5 and $\Phi_{S}=0.4$. Figure 6 presents the analysis of $C N$ as function of $q$ for various values of $k_{n, V}$ ranging from $10^{-5}$ to $10^{3} \mathrm{~N} / \mathrm{m}$ and for $k_{n, S}=10^{3} \mathrm{~N} / \mathrm{m}$. Additionally, this analysis was performed for $k_{n, S}=10^{2} \mathrm{~N} / \mathrm{m}$ and

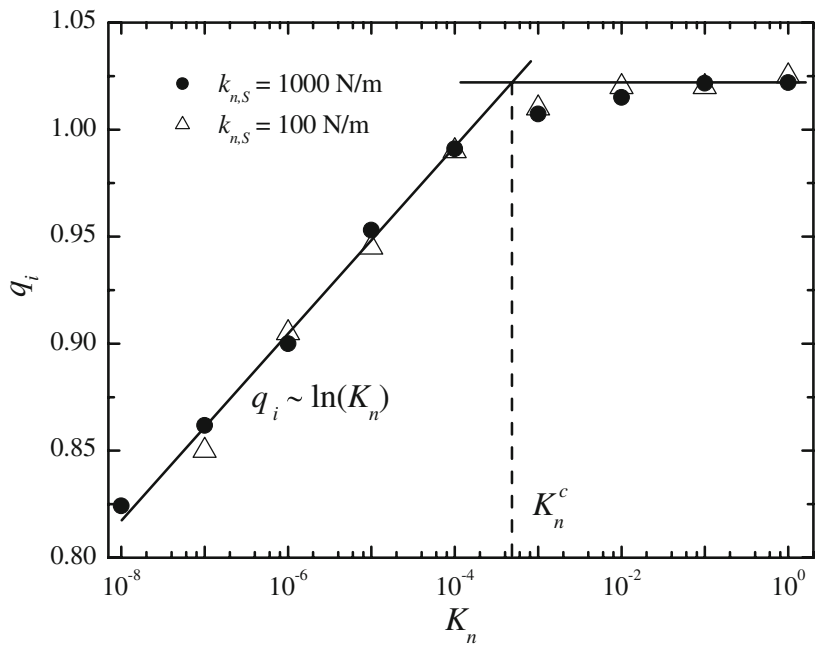

Fig. 7 Void- to structural particle radius ratio $q_{i}$ at the inflection point as a function of the void- to structural particle normal stiffness ratio $K_{n}$

$k_{n, V}$ ranging from $10^{-5}$ to $10^{2} \mathrm{~N} / \mathrm{m}$. In the following, the results are presented as function of the dimensionless voidto structural particle normal stiffness ratio $K_{n}=k_{n, V} / k_{n, S}$.

Two principal behaviors are observed for increasing voidparticle stiffness and thus for increasing $K_{n}$ : firstly, the inflection point is continuously shifted towards larger voidparticle sizes and secondly, the transition between region I and III becomes "sharper". These two observations will be elaborated in the following.

The shift of the inflection point towards larger void-particle sizes for increasing $K_{n}$ is summarized in Fig. 7, showing the continuous increase of $q_{i}$ for rising $K_{n}$. In particular, virtually identical curves are obtained for the two distinct values of $k_{n, S}$. For small normal stiffness ratios up to $K_{n}=10^{-4}$ the values for $q_{i}$ as function of $K_{n}$ follow a logarithmic law given in Eq. (10).

$q_{i}=0.02 \ln K_{n}+1.16$

The fit is very good as indicated by a correlation coefficient $R^{2}=0.993$. For $K_{n}>10^{-4}, q_{i}$ levels off at approximately $q_{i}=1.022$, which corresponds to a total volume fraction of 0.61 , close to the RCP volume fraction value.

The characteristic void- to structural particle normal stiffness ratio at which the transition from the logarithmic law to a constant value occurs is given by the intersection point of the respective fits. This value is termed $K_{n}^{c}$ and is found at $5.4 \times 10^{-4}$.

A sharper, i.e. more "step"-like, transition between region I and III is observed in Fig. 6 for increasing $k_{n, V}$. Mathematically, the "sharpness" of the transition expresses in an increasing slope of the curves at their inflection point, i.e. a higher value of $\Delta C N /\left.\Delta q\right|_{i}$, as summarized in Fig. 8 as function of $K_{n}$. As in the case of $q_{i}\left(K_{n}\right)$ identical curves are 


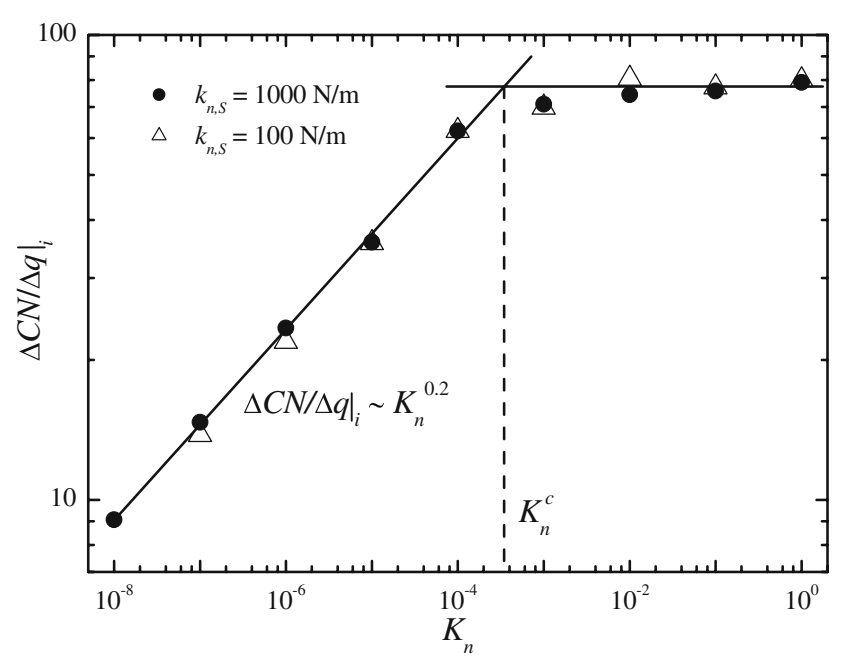

Fig. 8 Slope of the $C N(q)$-curves at the inflection point $\Delta C N /\left.\Delta q\right|_{i}$ as a function of the void- to structural particle normal stiffness ratio $K_{n}$

obtained for the two values of $k_{n, S}$. The values of $\Delta C N /\left.\Delta q\right|_{i}$ can be very well fitted against the void- to structural particle normal stiffness ratio $K_{n}$ up to $10^{-4}$ using a power law (Eq. 11).

$\Delta C N /\left.\Delta q\right|_{i}=410.3 K_{n}^{0.2}$

The correlation coefficient is $R^{2}=0.997$. For $K_{n}$-values higher than $10^{-4}$ the $\Delta C N /\left.\Delta q\right|_{i}$-values level off.

The small exponent of 0.2 might also suggest a logarithmic dependence between $\Delta C N /\left.\Delta q\right|_{i}$ and $K_{n}$, however, the logarithmic fit is of considerably lower quality $\left(R^{2}=0.91\right)$ compared to the power law fit.

The characteristic kink is found at $K_{n}^{c}=3.5 \times 10^{-4} \mathrm{~N} / \mathrm{m}$, which approximately corresponds to the value found for $q_{i}\left(K_{n}\right)$.

Equations (10) and (11) describe the $K_{n}$-dependence of $q_{i}$ and $\Delta C N /\left.\Delta q\right|_{i}$, respectively, for $K_{n} \leq 10^{-4}$. These equations allow to express $\Delta C N /\left.\Delta q\right|_{i}$ as function of $q_{i}$ yielding a relation of the form $\Delta C N /\left.\Delta q\right|_{i} \sim \exp \left(q_{i}\right)$. Indeed, an exponential fit gives a very high correlation coefficient $\left(R^{2}=0.992\right)$. The integration of $\Delta C N /\left.\Delta q\right|_{i}\left(q_{i}\right)$ allows to predict the function $C N_{i}\left(q_{i}\right)$, which also results in an exponential function. The fit of the simulated data using this function is good as well, achieving a correlation coefficient of $R^{2}=0.93$.

\section{Summary and conclusions}

In this paper, we presented VEM which allows for an efficient and fast computational generation of porous microstructures using DEM. The development of VEM was inspired by the experimental generation of heterogeneous colloidal microstructures using ASP. VEM is a stochastic method in opposition to earlier used BD simulation, in which the physical processes during the coagulation of the colloidal suspension were simulated. This numerical description of physical processes however requires much computing time. Thus, from a computational viewpoint, VEM is less intensive than BD simulation as VEM only includes a linear elastic contact law and damping.

The void expansion method permits to investigate the evolving microstructure as a function of the void particle size. In order to characterize the microstructure we used the mean coordination number. For all simulation parameters used throughout this research, the mean coordination number as function of the void- to structural particle radius ratio exhibits the same characteristic "step"-shape: a small slope with low mean coordination number in an initial stage, a transition stage with a sharp increase of the slope and, in a final stage, a small slope with high mean coordination number. The transition can be seen as a phase change between an initial stage, in which the particles can move freely and a final stage, in which the particles' movements are arrested. In this final stage, the particles are jammed and any further swelling of the void-particles corresponds to an increase in the strain energy in the structure. The inflection point in the transition stage characterizes the obtained simulation curves and enables a comparison between the various curves.

The sensitivity study of the mean coordination number by variation of the void-particle number, the structural particle number, the targeted volume fraction and the stiffness of the void-particles leads to the following results:

1. The variation of the void- and structural particle numbers and of the volume fraction of the structural particles reveals an inflection point at a constant total volume fraction of approximately $62 \%$, slightly below the RCP limit.

2. The total volume fraction of $62 \%$ constitutes an upper boundary for $\Phi_{S}$ and therefore for VEM.

3. The mean coordination number as function of the voidto structural particle radius ratio depends on the voidto structural particle number ratio alone and was found independent of the system size.

4. Structures with volume fractions ranging from 0.2 to 0.55 were successfully simulated using VEM. In particular, volume fractions above 0.4 were reached, which is crucial for a further simulation of the uniaxial compression of colloidal structures using DEM.

5. Above the inflection point, the mean coordination number as function of the total volume fraction follows a power law with exponent $\beta=0.37 \pm 0.04$.

6. An increasing void- to structural particle stiffness ratio $K_{n}$ reveals a twofold influence on the evolution of the mean coordination number as a function of the void- to structure-particle radius ratio: firstly, the inflection point 
is shifted to higher void- to structural particle radius ratios and secondly, the slope at the inflection point is increased.

7. For small $K_{n}$, the particle radius ratio $q_{i}$ and the maximum slope $\Delta C N /\left.\Delta q\right|_{i}$ at the inflection point are nicely fitted versus $K_{n}$ using a power and a logarithmic law, respectively. For $K_{n}$ approaching 1 the curves level off. The transition from a power and logarithmic law, respectively, to a constant value is found at a constant void- to structural particle normal stiffness ratio $K_{n}^{c}$ of approximately $4.5 \times 10^{-4}$. In particular, the curves $q_{i}\left(K_{n}\right)$ and $\Delta C N /\left.\Delta q\right|_{i}\left(K_{n}\right)$ do not depend on the structural particle stiffness.

The computational time needed to generate a VEM structure is determined by the number of void radius blow-up steps that are necessary to densely pack the structural particles. The number of void-particle blow-up steps essentially depends on $n$ and $\Phi_{S}$ and is increasing for decreasing $n$ or $\Phi_{S}$. Thus, smaller values of $n$ or $\Phi_{S}$ result in larger computational times. The simulations have further shown that for decreasing void-particle stiffness, less void blow-up steps are needed in order to reach the inflection point. However, for $k_{n, V}<0.001 k_{n, S}$ lower packing densities at the inflection point are obtained for decreasing void-particle stiffness. In $\mathrm{PFC}^{3 \mathrm{D}}$, the time-step essentially depends on the particle's mass and its stiffness as $\sqrt{m_{P} / k}$, where $m_{P}$ is the smallest particle mass and $k$ the largest particle stiffness in the system. Hence, for given particle densities the time-step is determined by the structural particle normal stiffness $k_{n, S}$ as long as $k_{n, V} \leq k_{n, S}$. For $k_{n, V}>k_{n, S}$, the time-step decreases resulting in longer simulation times.

To summarize, VEM allows for an efficient computational generation of porous microstructures over a wide range of volume fractions and the various relations analyzed in this paper predict the influence of the VEM simulation parameters on the microstructures. Further research comprises the influence of inter-particle friction, which in this study was set to zero in order to facilitate at most any particle rearrangements during the expansion of the void-particles.

We use VEM for an efficient generation of porous colloidal microstructures over a wide range of volume fractions for subsequent simulation of the mechanical properties using DEM. Towards this goal, the VEM-microstructures have to withstand the comparison with experimentally determined microstructures using for example confocal laser microscopy [18] or with structures obtained by other simulation techniques accurately describing the physical processes during the coagulation such as BD simulations [6, 7]. In this respect, an agreement in the mean coordination number is a necessary but not a sufficient condition. Additionally, further structural characterization methods, such as the pair correlation function [6], the common neighbor distribution function [19] or the recently introduced straight path distribution [20] must be considered in order to quantitatively compare the microstructures obtained by VEM to those obtained experimentally or using other computational techniques.

\section{References}

1. Wyss, H.M., Deliormanli, A.M., Tervoort, E., Gauckler, L.J.: Influence of microstructure on the rheological behavior of dense particle gels. AIChE J. 51(1), 134-141 (2005)

2. Gauckler, L.J., Graule, Th., Baader, F.: Ceramic forming using enzyme catalyzed reactions. Mater. Chem. Phys. 61, 78-102 (1999)

3. Tervoort, E., Tervoort, T.A., Gauckler, L.J.: Chemical aspects of direct coagulation casting of alumina suspensions. J. Am. Ceram. Soc. 87(8), 1530-1535 (2004)

4. Wyss, H.M., Tervoort, E., Meier, L.P., Müller, M., Gauckler, L.J.: Relation between microstructure and mechanical behavior of concentrated silica gels. J. Colloid Interface Sci. 273, 455-462 (2004)

5. Hesselbarth, D., Tervoort, E., Urban, C., Gauckler, L.J.: Mechanical properties of coagulated wet particle networks with alkali-swellable thickeners. J. Am. Ceram. Soc. 84(8), 1689-1695 (2001)

6. Hütter, M.: Local structure evolution in particle network formation studied by brownian dynamics simulation. J. Colloid Interface Sci. 231, 337-150 (2000)

7. Hütter, M.: Brownian dynamics simulation of stable and of coagulating colloids in aqueous suspension. Ph.D. thesis no. 13107, ETH Zurich, Switzerland (1999)

8. Russel, W.B., Saville, D.A., Schowalter, W.R.: Colloidal Dispersions. Cambridge University Press, Cambridge (1989)

9. Wyss, H.M., Hütter, M., Müller, M., Meier, L.P., Gauckler, L.J.: Quantification of microstructures in stable and gelated suspensions from cryo-SEM. J. Colloid Interface Sci. 248, 340-346 (2002)

10. Cundall, P.A., Strack, O.D.L.: A discrete numerical model for granular assemblies. Géotechnique 29, 47-65 (1979)

11. $\mathrm{PFC}^{3 \mathrm{D}}$ : User's Manual. Itasca Consulting Group, Inc., Minneapolis, Minnesota, USA (1995)

12. Brown, E.T.: Analytical and Computational Methods in Engineering Rock Mechanics, edn. Allen \& Unwin, London (1987)

13. Jaeger, H.M., Nagel, S.R.: Physics of the granular state. Science 255(5051), 1523-1531 (1992)

14. Wyss, H.M., Tervoort, E.V., Gauckler, L.J.: Mechanics and microstructures of concentrated particle gels. J. Am. Ceram. Soc. 88(9), 2337-2348 (2005)

15. Kruyt, N.P., Rothenburg, L.: Statistics of the elastic behaviour of granular materials. Int. J. Solids Struct. 38, 4879-4899 (2001)

16. O'Hern, C.S., Silbert, L.E., Liu, A.J., Nagel, S.R.: Jamming at zero temperature and zero applied stress: the epitome of disorder. Phys. Rev. E 68, 011306 (2003)

17. Zhang, H.P., Makse, H.A.: Jamming transition in emulsions and granular materials. Phys. Rev. E 72, 011301 (2005)

18. Crocker, J.C., Grier, D.G.: Methods of digital video microscopy for colloidal studies. J. Colloid Interface Sci. 179, 298-310 (1996)

19. Clarke, A.S., Jónsson, H.: Structural changes accompanying densification of random hard-sphere packings. Phys. Rev. E 47(6), 39753984 (1993)

20. Schenker, I., Filser, F.T., Aste, T., Gauckler, L.J.: Microstructures and mechanical properties of dense particle gels: microstructural characterization. J. Eur. Ceram. Soc 28(7), 1443-1449 (2008) 